\title{
REFLECTA - Artificial Intelligence Based Smart Mirror
}

\author{
Chethan K, Adnan Ahmed, Nikhil Ganapathy, Pragathi N Simha, Sourabh Kothari
}

\begin{abstract}
This paper interprets the development of an artificial intelligence-based mirror that shows an inconspicuous network interface for an ideal home setting. The mirror concedes an interaction through which the users can have an access and control to the smart appliances present at homes and also an ingress to personalized services. Priority is given to ensure comfort in accessing the basic services with a minimum hinder to user's mediation. Taking an example, the face recognition feature is used to identify the user that faces the mirror and provide a module-based interface to access user data such as calendar, email, phone, social media, and other services. Reflecta basically works on Raspberry Pi 3 Model B board. It acquires a service-oriented architecture and is developed to use various utilities. The mirror, the appliances, data and news feeds use web services and cloud platform. Reflecta's functionalities have been exhibited by developing an easily accessible home automation system based on ESP node service that simplifies the integration of home appliances and various customized web and internet services.
\end{abstract}

Index Terms-Face recognition, Raspberry pi 3, ESP node service, Home Automation.

\section{INTRODUCTION}

The standard and quality of life is changing vigorously by the help of interactive computing and embedded systems that are being used in our daily life. A variety of devices and products based on this interactive technology have been introduced to the world. With this kind of artificial intelligence and interactive computing, we are provided with a comfortable, user convenient and a very secure personal service. Whether it is a home or a workspace, it makes all the users pretty convenient to work or enjoy the multimedia. We use the mirror multiple times every day to see if we have dressed well or how our hair looks and even do a lot of grooming in front of it. Reflecta is an advancement effort to develop an embedded intelligence onto a mirror and offer enhanced features such as latest news, headlines, weather, and local time corresponding to the user's location. The Smart Mirror is a stepping stone in development of smart homes with the help of embedded artificial intelligence. It's found its applications in various workspace as well. This

Revised Manuscript Received on April 12, 2019.

Chethan K,Department of Electronics and Communication Engineering, Vidyavardhaka College of Engineering, Mysuru, Karnataka 570002. India. (E-mail: chethank@ vvce.ac.in)

Adnan Ahmed, Department of Electronics and Communication Engineering, Vidyavardhaka College of Engineering, Mysuru, Karnataka 570002. India (E-mail: adnanhasham20@gmail.com)

Nikhil Ganapathy, Department of Electronics and Communication Engineering, Vidyavardhaka College of Engineering, Mysuru, Karnataka 570002. India (E-mail: nikhilganapathy007@gmail.com)

Pragathi N Simha,Department of Electronics and Communication Engineering, Vidyavardhaka College of Engineering, Mysuru, Karnataka 570002. India (E-mail: pragathi.n.simha@gmail.com)

Sourabh Kothari, Department of Electronics and Communication Engineering, Vidyavardhaka College of Engineering, Mysuru, Karnataka 570002. India (E-mail: sourabh.enviox@gmail.com) project intends to implement an electronic board known as RASPBERRY PI 3 board. Basically, the Smart Mirror consists of a 2-way mirror with a hardware technology including the LCD display and raspberry pi 3 board connected to process the inputs and display the outputs in the LCD screen which tend to appear in the mirror.

\section{LITERATURE REVIEW}

Philips, in 2003, unveiled a product called Mirror TV. It was developed using similar foundation of the current smart or interactive mirrors. The product was basically 2-way mirror placed in front of a normal Television so that it would appear as a reflective mirror when turned off and when turned on, it would work as a normal television.

Philips, again in 2005, released a research project called My-Heart. It was a mirror that showed vital information about the patient. Unlike the Mirror TV, My-Heart would integrate a display under a mirror which displayed various information and medical statistics.

James Law Cybertecture developed a Smart mirror in 2011 that was sold commercially. The mirror was something very similar to the smart mirrors we have today. It consisted of an LCD-display (32 inches) which was behind a 2-way mirror (37 inches). The screen could show various information such as time, weather, and custom widgets. It could also stream the internet or work as a Television whenever wanted. It had a lot of input devices such as a phone application, remote and also an on-screen keyboard.

Microsoft, in 2016, released details regarding an interactive mirror they were developing. The objective wasn't to sell it commercially but was to help everyone build it. They published all the information to build a Smart Mirror and released the source code on a repository on Github which was available to everyone. Users could now build their own mirror as a personal Do-It-Yourself project.

At the 2017 CES Convention, Griffin Technologies showcased their work on a concept of Smart Mirror. The product was called "The Connected Mirror". The company built a lot of smart home appliances which be connected to the mirror and it would work as a hub from where everything at home could be controlled.

Recently there have been number of reports on Smart Mirror and its applications. Smart mirrors have been used as E-health assistant [1], Health Monitoring [2], Healthy Lifestyle [3], Smart Home [4], in IOT based applications [5], and as a two way mirror and display system [6]. 


\section{PROBLEM STATEMENT}

The recent world has become a place of an intense competition among the people. Human race has become more goal oriented and strive to be the best in all aspects whether its sports, business or entertainment. We all, ultimately, strive to be the best. Following the news, adapting the varied weather conditions are some of the interruptions that hinder our daily progress. Activities like these consume a lot of our time and can be very distracting which might affect our day-to-day activities.

People value their appearance greatly and spend a ridiculous amount of time in front of a mirror throughout their daily routine. And this is the exact time where a lot of important things can be performed. But spending time with a smartphone, managing daily tasks, while preparing for the day would be a hard task. Therefore, a device with certain technology is required which allows a person to efficiently complete all the work needed for them to prepare for the day. And all of this has to be done at the same time and in one place. Hence, an Artificial Intelligence based Smart Mirror.

The objective of Reflecta is to provide an interactive display with the simplest interface to access various information that is needed to prepare well for the day. By using a 2-way mirror and an LCD display information like weather, time, calendar, news, voice controlled AI, YouTube, home automation service and many more functions can be performed simultaneously together.

\section{METHODOLOGY}

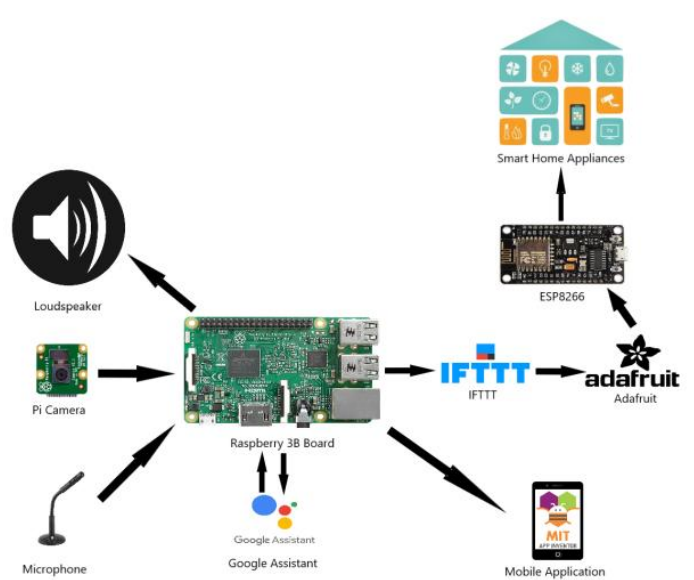

Fig.1 - Block Diagram of Reflecta's Interface

The block diagram of Reflecta's hardware interface is shown in Fig. 1. Itmainly consists of Raspberry Pi 3, an LCD display, an ESP8266 module, A camera, PIR and DTH sensors and a smartphone application that are placed respectively as shown.

\section{A. HARDWARE DESIGN}

Reflecta uses the following Hardware Components:

\section{a) Raspberry Pi 3:}

The Raspberry Pi 3 Model B is a very small sized computer. By providing a power supply, I/O devices, a micro SD card which has an installed Linux based Operating System in it, we get a fully developed computer which can run various applications. The board has a built in Bluetooth and WiFi connectivity, which makes it a perfect device that is IOT-ready. Raspberry Pi 3 Model B Board has 4 USB 2.0 ports, 40 GPIO pins and a micro SD card slot for memory. The Board needs a power supply of $5 \mathrm{~V}$ with 2 amps minimum, to function properly. Keyboard and Mouse can be connected using any of the USB ports available. The board also has an HDMI port through which HD output can be sent to a monitor/TV which will work as a display. The operating system should be pre-installed on a micro SD card.

\section{b) 2-Way Mirror:}

A 2-Way mirror is a complementary mirror which is moderately transitive and somewhat reflective.

\section{c) ESP8266 Node MCU (ESP-12E):}

The Node MCU is an open-source development \& firmware board. It helps us build an IOT product with a platform like Arduino IDE or just in few lines of Lau script. It comprises of a hardware that is established on the grounds of ESP-12 module with a firmware that runs on the Wi-Fi SoC ESP8266. The ESP8266 Wifi Module is a SoC that has an integrated TCP/IP protocol stack which gives various microcontrollers an access to any desired Wi-Fi network. The ESP8266 is capable enough to either host an application or offload all Wi-Fi network functions from another application processor.

\section{d) LED Display Panel:}

All the displays in Laptops and other computers comprises of a technology called LED (Light Emitting Display). Like gas-plasma and LED technologies, the LEDs are responsible for the displays to be really thin. Now because they basically block the light rather than emitting it, the LEDs consume much less power than other display technologies.

e) Sensors:

1. PIR Sensor: Infrared rays are measured by a passive infrared sensor (PIR sensor). They are most often used in motion detectors as they measure the rays that are radiated from the objects in its field of view.

2. DHT Sensor: The DHT sensors measure the humidity and temperature of the surroundings. It consists of a capacitive humidity sensor and a thermistor. The sensor has an inbuilt ADC converter that converts Analog to Digital signal and gives us a digital reading of humidity \& temperature.

\section{f) Microphone:}

A microphone, colloquially a transducer that converts sound into an electrical signal.

\section{g) Smart Home Appliances:}

Smart home refers to the modern houses which have various lightings, appliances, and electronic devices that can be remotely controlled by the owner, often via a mobile app or a web application.

Published By: 


\section{B. SOFTWARE DESIGN}

Reflecta uses the following Softwares:

a) Raspbian:

Raspbian is the main operating system for Raspberry Pi. It is a Debian-based Linux OS. Raspbian comprises over 35,000 packages for easy installation on the Raspberry $\mathrm{Pi}$ board.. It comprises a modified LXDE desktop environment and an Open box stacking window manager.

\section{b) Python:}

Python is a high-level programming language used for general purpose programming. Python features an automatic memory management and a dynamic type system.

c) CSS:

Cascading Style Sheets is a style sheet language which describes a document's presentation that is written in a language like HTML. CSS is a foundation technology of the World Wide Web, along with HTML and JavaScript.

d) Node.js:

Node.js is a platform built on Chrome's JavaScript runtime for easily building fast and scalable network applications. Node.js uses a non-blocking I/O model which is event- driven. It is lightweight and efficient.

\section{e) Arduino IDE:}

Arduino IDE (Integrated Development Environment) is the platform where codes can be written, compiled and forged on the boards with microcontrollers.

\section{f) MIT App Inventor:}

It is the easiest tool that allows users to build software applications for the Android OS.

g) IFTTT:

If This Then That, also known as IFTTT is a free webbased service where we can create applets, which are chains ofsimple conditional statements.

\section{OPERATIONS}

\section{1) Reflecta as a Natural Mirror Interface:}

Its works as a plane reflective mirror and can be used as the one to do our daily work in front of. This is because of the high concentration of aluminum content on one side of the 2-Way mirror.

\section{2) Reflecta as a Real Time Data Display System:}

It is used to display the current date, time, calendar, weather updates, and temperature of the room, current news feed, scheduled reminders, traffic update and can also keep in track of your daily activities.

\section{3) Reflecta as a Personal Assistant:}

It updates one's personal messages, emails, social media notifications, meetings and appointments, to do list and also stores down private information provided by the user. It basically identifies the user using face recognition technology to provide the personal information to the particular user. It can also book an app based cab like Uber or Ola for the user.

\section{4) Reflecta as an AI Voice Controlled Interaction:}

It can interact with the user and can provide all the necessary information to the user by accessing the information through internet. It also includes inbuilt voice commands for daily interactions.

\section{5) Reflecta as a Smart TV:}

It can access and stream media from various platforms such as Youtube, Spotify, Soundcloud, etc.

\section{6) Reflecta as a Home Automation Hub:}

It can have an access and control all the smart appliances at home and can also activate or turn on the devices at a desired time set by the user.

\section{7) Reflecta as a Security System:}

It can have an access to security cameras and display it to the user. It also clicks the picture of an unknown face trying to access the mirror.

\section{ALGORITHM}

\section{1) Reflecta as an Information Display System}

Step 1: Connect the Raspberry pi board with the display and design framework using raspbian and other software as mentioned above.

Step 2: Get information such as time, date, weather and all the other respective details as mentioned above.

Step 3: All the respective information such as weather, news etc. can be obtained by linking with API.

\section{2) Reflecta as a Personal Assistant}

Step 4: Setup a web camera in front of a mirror and connect it to the raspberry pi board.

Step 5: Write down the code for face detection in the raspbian software.

Step 6: Check if the face detection is taking place and with it the probability of the face detection.

Step 7: Provide the personal information such as messages, emails etc. by linking it with the social media API's.

\section{3) Reflecta as a Home Automation Hub}

Step 8. Link the raspberry pi with firebase using IFTTT to perform Home automation for the smart home appliances.

Step 9: Build an app using the MIT app inventor to have access to smart devices using smartphone.

\section{4) Reflecta as an AI Voice Controlled Interaction:}

Step 10: Make Reflecta work as voice controlled AI device by going deep into machine learning and artificial intelligence. 


\section{WORKING PRINCIPLE}

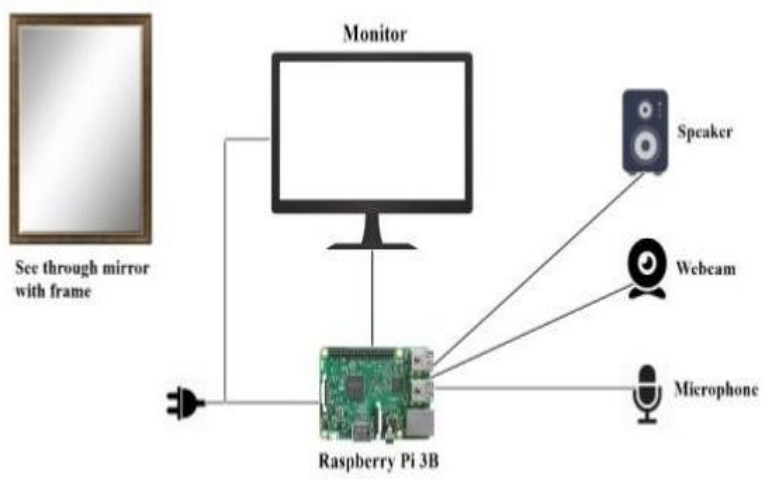

Fig.2 - Basic Architecture of Reflecta

A pictorial representation of Reflecta's architecture is shown in Fig.2. First, a frame for the mirror is built and attached to the 2-way see through mirror. Front side of the frame is for the viewing of user and on the other side is where all the components are. An LED is attached to display with the frame and connect the LCD display with the raspberry pi via HDMI cable. USB microphone and USB web were then connected with the raspberry pi. Finally, the power source for both the raspberry pi and LCD display was established.

As the raspberry pi has its own operating system, the Raspbian operating system was booted for the project into the raspberry pi. It was updated and upgraded to increase the CPU speed. The default version of the operating system consisted of an older version of the Node which did not consist of NPM. So, the Node was reinstalled which included the NPM. Next, pip was installed which was a package installer of python and it helped to install numerous packages. However, python Speech Recognition was installed as it was used to convert audio into texts and for processing the video inputs. An OpenCv, a library of programming functions was used which focused on real time computer vision.

Now moving to the coding and designing part, HTML, CSS and JavaScript were used to display the information on the LCD display in such a way that only the information's would appear before the user. And for the AI and other modules, python programming language was used. However, an open source AI library was used to train our AI.

The key features of our design are:

(1) Time and Date: The time of the CPU used (Raspberry $\mathrm{Pi}$ ) in the mirror was shown

(2) Calendar: The international calendar in the mirror as well as the upcoming holidays was integrated in the system. The help of open source website to fetch the API of the calendar was taken.

(3) News: The news functionality was integrated, which will show the RSS feed of any newspaper of the world.

(4) Artificial Intelligence (AI): Figure 3 shows the working principle of AI.

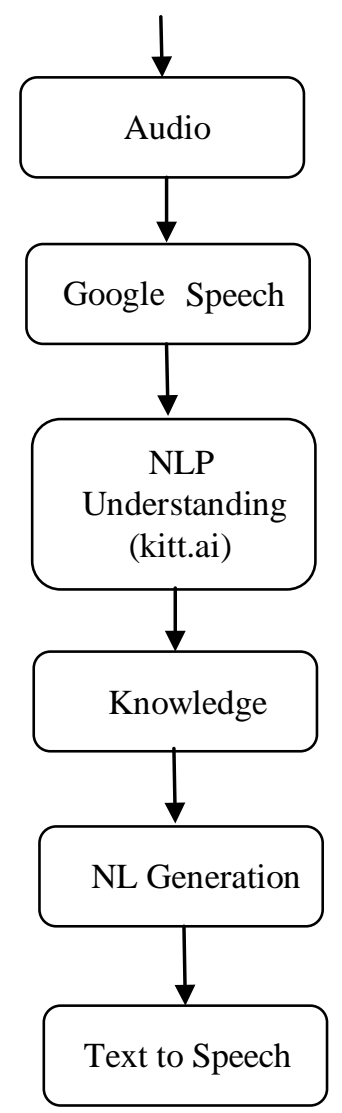

Fig.3 - Working Principle of AI

AI will take voice information as input and through voice recognition, will identify users. Processing audio or speech is more time consuming than processing text. So, the audio input will be converted into text through speech software, here used google speech to perform this task. To make AI understand the text, wit.ai was used which would have intent and entity. Then the AI needed to retrieve relevant information as per user request and this the knowledge phase was called. Once the information is obtained, AI needs to form a complete sentence to express the information, so this is done in the Natural Language Generation state. The AI has a complete sentence, this text must be converted into speech. Thus, this loop will be continued.

\section{PERFORMANCE EVALUATION\& RESULTS}

After setting up all the hardware and software components, the model was checked for the outcome. The first launch wasn't successful as the AI wasn't taking any voice command due to the failure of speech recognizer package. However, the library and package were reinstalled and then launched the model again. This time, it could recognize the voice, but the model was responding very slowly.

Next, the problem we faced launching the system was to make the AI understand the different types of entities, as the app was trained under wit.ai. The different entities in every launch was created as the amount of voice command as well as understanding level of AI was increasing. So, some fixed entities were added to enrich knowledge of the AI.

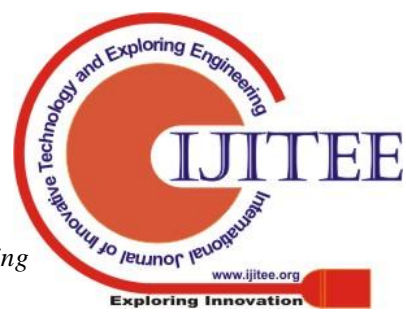


Furthermore, the model was not fast enough as the RAM of the Raspberry Pi was only 1.0 GB and the processor was only $1.2 \mathrm{GHz}$. However, overclocking the pi processor was tried and came up with a good result. The GPU was decreased to get the maximum amount of speed from the Raspberry Pi.

\begin{tabular}{|l|l|l|}
\hline $\begin{array}{l}\text { CPU Speed } \\
\text { (arm speed) } \\
(\mathbf{C C} / \mathbf{s})\end{array}$ & $\begin{array}{l}\text { GPU Speed } \\
(\mathbf{C C} / \mathbf{s})\end{array}$ & $\begin{array}{l}\text { Efficiency } \\
(\boldsymbol{\%})\end{array}$ \\
\hline 800 & 126 & 80 \\
\hline 1100 & 126 & 85 \\
\hline 1350 & 16 & 90 \\
\hline
\end{tabular}

\section{Table 1 - Observation of performance efficiency in} different clock speed

Moreover, the data were demonstrated in the table was provided by calculating the CPU usage in different clock speed. In minimal GPU speed and maximum CPU speed the model worked perfectly.

\section{CONCLUSION}

At the end we build a working prototype of a smart mirror called Reflecta which can have access to all the smart home appliances, lightings, etc. It has a great potential in enhancing the experience of a user to access vital information from various places and also interact with the user data and information available from the internet. It allows users to see relevant information effortlessly. It saves a lot of effort and time that is needed to access such information by displaying all in one place. An infrared touch screen technology can be added in the future to make Reflecta better and more interactive.

\section{ACKNOWLEDGEMENT}

The authors express gratitude towards the assistance provided by Accendere Knowledge Management Services Pvt Ltd for the manuscript preparation.

\section{REFERENCES}

1. B. Cvetkoska, N. Marina, D. C. Bogatinoska and Z. Mitreski, "Smart mirror E-health assistant — Posture analyzealgorithmproposedmodelforupright posture," IEEE EUROCON 2017 -17th International Conference on Smart Technologies, Ohrid, 2017, pp. 507-512

2. HamidurRahman, Shankar Iyer, Caroline Meusburger, KoljaDobrovoljski, MihaelaStoycheva, VukanTurkulov, Shahina Begum, and MobyenUddin Ahmed, "SmartMirror: An Embedded Non-contact System for Health Monitoring at Home," HealthyIoT 2016, LNICST 187, 2016, pp. 133-137

3. Sara Colantonio, Giuseppe Coppini, DanilaGermanese, Daniela Giorgi, Massimo Magrini, Paolo Marraccini, Massimo Martinelli* , Maria Aurora Morales, Maria AntoniettaPascali, Giovanni Raccichini, Marco Righi, OvidioSalvetti, "A smart mirror to promote a healthy lifestyle," Biosystems Engineering, 138, 2015, pp 33-43.

4. Chidambaram Sethukkarasi, VijayadharanSuseelaKumariHariKrishnan,

KaruppiahPalAmutha, Raja Pitchiah, "Interactive Mirror for Smart Home," Int J on Adv in Intell Systems, 9, 2016, pp 148 160
5. M. M. Yusri et al., "Smart mirror for smart life," 2017 6th ICT International Student Project Conference (ICT- ISPC), Skudai, 2017.

6. Derrick Gold, David Sollinger and Indratmo, "SmartReflect: A modular smart mirror application platform," 2016 IEEE 7th Annual Information Technology, Electronics and Mobile Communication Conference (IEMCON), Vancouver, 2016 